\title{
Two Clinical Cases of Metastatic Merkel Cell Carcinoma Illustrating Avelumab Tolerability in Elderly Patients
}

\author{
Bridget Josephs ${ }^{1}$ Sanjeev Sewak ${ }^{2}$ \\ ${ }^{1}$ Medical Oncology Advanced Trainee at Launceston General Hospital, \\ Tasmania, Australia \\ 2 Peninsula and South East Hematology and Oncology, Melbourne, \\ Australia \\ Ind J Med Paediatr Oncol 2021;42:599-602.
}

Address for correspondence Sanjeev Sewak, MBBS, FRACP, Peninsula and South East Hematology and Oncology, Melbourne, VIC 3199, Australia (e-mail: sewaks10@gmail.com).

\section{Introduction}

Merkel cell carcinomas (MCCs), typically appearing as a painless pearly skin nodule that can be red or violaceous in color, carry with them a high propensity to both recur locally and to metastasize. Estimated incidence ranges from 0.2 to 1.6 per $1,00,000$ people per year, depending on geographical location. The highest reported rates are in Queensland, Australia.,2 Prognosis in metastatic disease is classically poor, with 5-year overall survival in the order of 0 to $18 \%{ }^{1}$ The mean age at diagnosis is 74 and 76 years, respectively, in males and females. ${ }^{3}$

Tumor genesis is believed to be due to Merkel cell polyomavirus virus infection in 60 to $80 \%$ of cases, and/or exposure to ultraviolet radiation with resultant mutagenesis. ${ }^{1,4}$ Chronic immunosuppression, another risk factor for this malignancy, also ties in with immune dysregulation. ${ }^{1}$
Neoantigens produced by the tumor cells are expressed on the cell surface and are recognized by the T cells. Immune checkpoint inhibition leads to $\mathrm{T}$ cell activation and subsequent malignant cell killing.

\section{Case Reports}

\section{Case 1}

At the age of 95 years, a Caucasian man was initially diagnosed with a localized MCC in September 2016.

His medical history included atrial fibrillation, valvular heart disease, resected meningioma complicated by a stroke, legal blindness, glaucoma, gout, hypertension, and hyperlipidemia. He was an ex-smoker.

The primary skin lesion, located on the right lower limb, was excised at the time. Histopathology was consistent with a
DOI https://doi.org/ 10.1055/s-0041-1740949. ISSN 0971-5851.

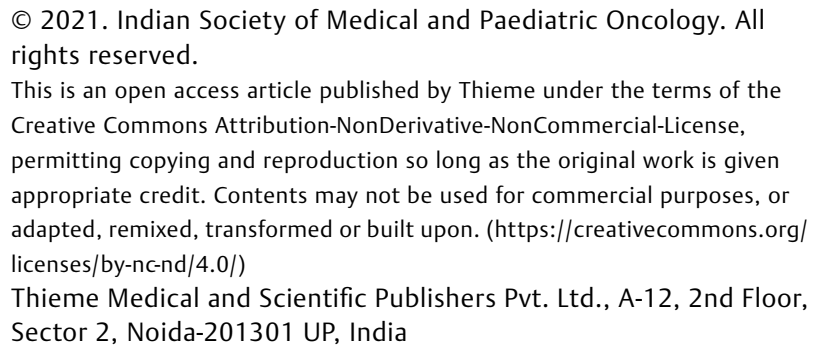

This is an open access article published by Thieme under the terms of the Creative Commons Attribution-NonDerivative-NonCommercial-License, permitting copying and reproduction so long as the original work is given appropriate credit. Contents may not be used for commercial purposes, or adapted, remixed, transformed or built upon. (https://creativecommons.org/ licenses/by-nc-nd/4.0/)

Thieme Medical and Scientific Publishers Pvt. Ltd., A-12, 2nd Floor, Sector 2, Noida-201301 UP, India 

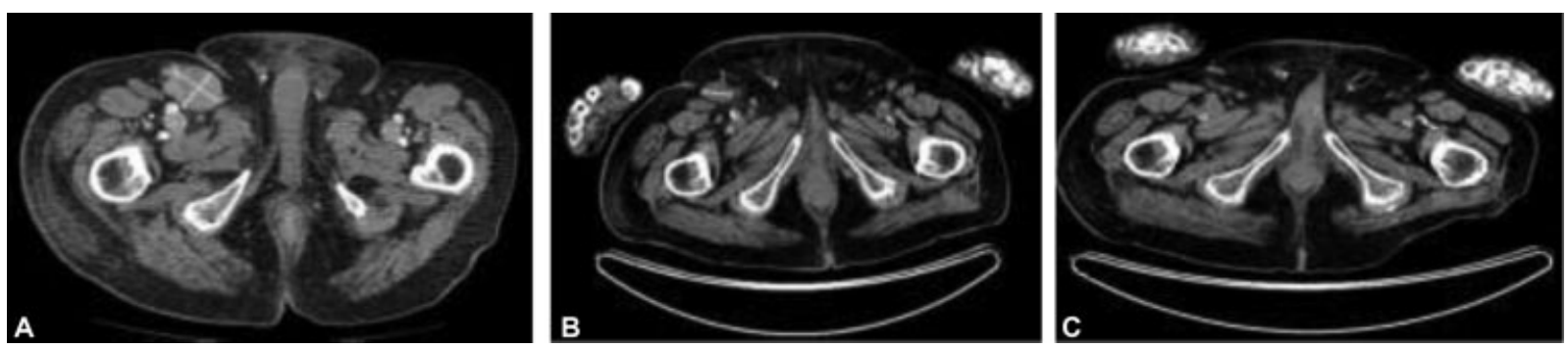

Fig. 1 Computed tomography (CT) showing (A) right pelvic lymphadenopathy at diagnosis in July 2017; (B) positron emission tomography computed tomography (PET/CT) restaging September 2017 postradiotherapy showing a partial response; (C) PET/CT September 2018 showing a complete response to avelumab.

$20 \mathrm{~mm}$ MCC with lymphovascular invasion but clear resection margins. Sentinel lymph node biopsy and adjuvant radiotherapy were not performed in view of his advanced age.

In June 2017, the patient presented with right groin lymphadenopathy and associated right leg edema. Imaging revealed extensive right inguinal, pelvic and paraaortic lymph node metastases, with the largest node measuring $38 \times 66 \mathrm{~mm}$.

He was treated with palliative radiotherapy to his right pelvis and groin, receiving a total dose of 28 Gray (Gy) in eight fractions. A positron emission tomography/computerized tomography (PET/CT) scan 6 weeks later in September 2017 demonstrated a partial response to treatment. In October 2017, he received avelumab at a dose of $10 \mathrm{mg} / \mathrm{kg} \mathrm{2-}$ weekly via the Merck Patient Access Scheme. His ECOG (Eastern Cooperative Oncology Group) performance status was 2 but improved to 1 with the ongoing response. The patient was followed clinically every 2 weeks with a blood test and physical examination of his edematous right leg.

The PET/CT performed 11 months later in September 2018 showed a complete metabolic response to avelumab (-Fig. 1). As of January 2021, the patient remained in clinical remission and continued on avelumab that he was tolerating well without any toxicity. Unfortunately, he had a mechanical fall in February 2021 and sustained a subdural hemorrhage (on CT scanning) and died peacefully at the age of 99.

\section{Case 2}

A 80-year-old Caucasian man was initially diagnosed with a localized left preauricular MCC that was excised in September 2017.

Past medical history included ischemic heart disease, hypertension, type-2 diabetes, and multiple previous skin cancers fully excised. His ECOG performance status was 0 .

The lesion required re-excision in October 2017 due to positive margins, and this was followed by a split skin graft. Subsequent histopathology confirmed MCC and showed lymphovascular invasion but clear margins. PET/CT scan at diagnosis was negative for metastatic disease. The patient then received postoperative adjuvant radiotherapy to the left preauricular and parotid regions as well as left upper neck at a total dose of $45 \mathrm{~Gy}$ in 20 fractions that he completed in December 2017.

In July 2018, the patient presented with left cervical lymphadenopathy. A PET/CT scan also demonstrated multiple bony metastases and two liver metastases. A core biopsy from a cervical node confirmed metastatic MCC. Palliative carboplatin and etoposide drugs were commenced in August 2018. Follow-up imaging post three cycles of chemotherapy in October 2018 revealed a partial response. The patient was then eligible for second-line avelumab 10 $\mathrm{mg} / \mathrm{kg}$ 2-weekly which he commenced in December 2018. A PET/CT scan performed 5 months later in April 2019 revealed a complete response (CR) (-Fig. 2). By December 2020, the patient had ceased avelumab and is in complete remission (on PET/CT scanning) from MCC. For pragmatic reasons, we decided with the patient's input to watch him closely off therapy. Coming to hospital every 2 weeks for his infusion was becoming onerous. Therapy can be reinstituted if he relapses. He remains in clinical remission when seen in July 2021.

He tolerated avelumab well apart from grade 2 infusion reaction (rigors) with the first two cycles of immunotherapy. Further infusion reactions were prevented with paracetamol, ranitidine, and promethazine premedication.

\section{Discussion}

MCC is a rare but aggressive neuroendocrine skin malignancy historically associated with a poor prognosis in advanced disease. While initially a chemo-sensitive tumor, responses are typically short-lived. Surgery is reserved for patients with primary MCC without metastases. Radiotherapy can be used as adjuvant therapy or for palliation of symptoms. Immune checkpoint inhibitor therapy has demonstrated promising durable antitumor activity for this malignancy
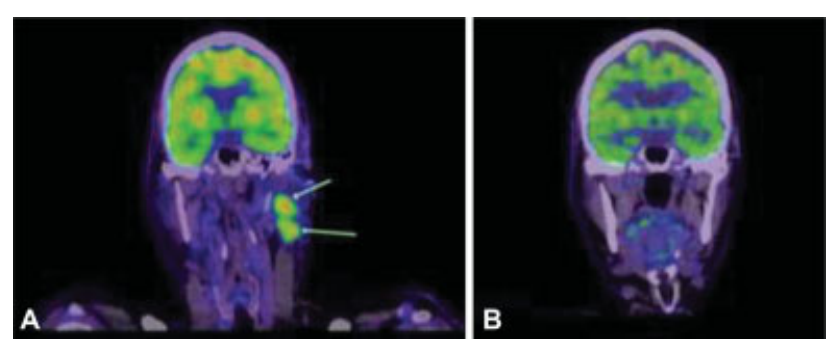

Fig. 2 Positron emission tomography (PET) scans showing (A) 18fluoro-2-deoxy-glucose avid left cervical lymphadenopathy at diagnosis of metastatic Merkel cell carcinoma in July 2018; (B) complete resolution of cervical lymphadenopathy in April 2019 following 5 months of treatment with second-line avelumab. 
in phase II trials. We have presented two patients with metastatic MCC treated with avelumab, a programmed death ligand (PD-L1) inhibitor.

These two cases illustrate the low toxicity profile of avelumab and the high response rates. In the JAVELIN Merkel 200 trial, the median age of the cohort was 72.5 years and age range was between 64.5 and 77 years. ${ }^{5}$ Our two patients were much older at the age of 80 and 95 when they started treatment with avelumab, illustrating its safe use in very elderly patients.

Until recently, the mainstay of treatment for metastatic MCC has been palliative chemotherapy with common regimes including carboplatin or cisplatin with etoposide or topotecan. ${ }^{1}$ Palliative radiotherapy is also an adjunct for local symptom control. While this type of cancer is typically considered to be chemosensitive, responses are usually only transient before cancer progression leading to a fatal outcome. Median progression-free survival (PFS) in the literature is reported to be only 3.1 to 4.6 months. ${ }^{6}$ Chemotherapy can also be associated with significant toxicity. Studies show that the median survival with chemotherapy alone is 9 months. The toxic death rate from chemotherapy in 1999 was $7.7 \%$ and maybe lower in 2021 with better supportive care. ${ }^{7}$ Consequently, there has been interest in pursuing novel agents to improve clinical outcomes for patients.

Immunotherapy with immune checkpoint inhibition is emerging as a promising new therapy in the treatment landscape for metastatic MCC. Preliminary data from several nonrandomized trials using immunotherapy in patients with metastatic MCC suggest durable responses when compared with chemotherapy with considerably lower toxicity. ${ }^{5,8}$ We monitored our patients for immune-related toxicities such as rash, diarrhea, hyperthyroidism, hypothyroidism, and hypopituitarism every 2 weeks.

Immunotherapy agents trialed have included PD-L1 and programmed cell death protein 1 (PD-1) directed therapies including avelumab, a PD-L1 inhibitor, and nivolumab and pembrolizumab, both PD-1 inhibitors. ${ }^{1,5,9,10}$ The mechanism of action of PD-L1 and PD-1 immune checkpoint inhibitors is to effectively enhance the immune systems antitumor effect by allowing $\mathrm{T}$ cell mediated destruction of tumor cells.

The pivotal JAVELIN Merkel 200 study (part A), involving 88 patients with metastatic MCC who had relapsed after at least one line of chemotherapy, demonstrated an overall objective response to avelumab of 33\% (29 patients). A CR occurred in $11.4 \%$ ( 10 patients). Of those $33 \%$ of patients who any response (complete/partial), in the latest update of this trial, the median duration of response after $\geq 36$ months of follow-up was 40.5 months. ${ }^{5,9,11}$ Case 2 had treatment as per the part A Javelin Merkel 200 study.

Case 1 had therapy as per part B of the study. Part B examines the use of avelumab in the first-line setting for metastatic MCC, and observed a trend toward higher response rates in chemotherapy-naive patients compared with patients who had already had prior lines of treatment. At interim analysis, after a median follow-up of 5.1 months, a response rate of $62 \%$ was achieved in the 39 patients enrolled with ongoing responses in $78 \%$ of this cohort. Additionally, avelumab was generally well tolerated with no grade 4 or higher treatment related adverse-events ${ }^{8}$.

The Keynote 017 phase II trial reported on 50 patients with advanced MCC who were naïve to systemic therapy. Pembrolizumab was given at a dose of $2 \mathrm{mg} / \mathrm{kg}$ every 3 weeks. The objective response rate was $56 \%$ with $24 \%$ of patients achieving a CR. The 2-year overall survival rate was $68.7 \%$. Pembrolizumab had fewer grade $3 / 4$ toxicities than avelumab (in $28 \%$ vs. $74 \%$, respectively). However, there was one treatment-related death in the Keynote 017 study and none in the Javelin Merkel 200 trial. $^{9,12}$

Recently, the Food and Drug Administration in the United States has approved the flat dosing of avelumab based on the pharmacokinetic studies published by Novakovic and colleagues. ${ }^{13}$ The approved dosing is $800 \mathrm{mg}$ avelumab every 2 weeks.

Until recently, there was no established standard of care or approved therapies for the treatment of metastatic MCC. Findings from the JAVELIN Merkel 200 study led to the pharmaceutical approval of avelumab for use in many countries, including Australia.

Our two case studies demonstrate an excellent $C R$ to avelumab with ongoing durable responses and no significant treatment-related adverse-events. Our study has limitations because of its small sample size. Even the largest trial (JAVELIN Merkel 200) had 88 patients that reflects the low incidence of this uncommon cancer.

Conventional treatment, palliative radiotherapy in case 1 and chemotherapy in case 2, produced partial responses. CRs were only achieved with avelumab. At the time of writing, PFS is 40 months in case 1 and 41 months in case 2, higher than reported in the JAVELIN clinical trials.

Other case reports in the literature also demonstrating complete, durable responses to immunotherapy in metastatic MCC, include O'Brien and Power reporting an ongoing 19 months PFS with pembrolizumab. ${ }^{10}$

There are now several ongoing trials testing immunotherapy in the adjuvant setting after wide local excision. These include the ADMEC-O study (NCT 02196961) testing the efficacy of adjuvant nivolumab in patients with completely resected MCC. The ADAM trial (NCT 03271372) looks at the efficacy of avelumab versus placebo in stage $3 \mathrm{~b}$ resected MCC. $^{14}$

\section{Conclusion}

In conclusion, we report two cases of metastatic MCC in which both patients have had complete and durable ongoing responses to avelumab. Side effects have been minimal.

Immunotherapy is emerging as an efficacious, revolutionary therapy for metastatic MCC, expanding our therapeutic artillery in this rare disease where previously therapeutic options had been limited.

\section{Authors' Contribution}

Both authors were involved in concept, design, definition of intellectual content, literature search, case report, manuscript preparation, editing, and review. 


\section{Patient Consent}

Formal patient informed consent was not required at our institution as the cases were anonymous. Ethics Committee Waiver was obtained for publication.

\section{Funding}

None.

\section{Conflict of Interest}

None declared.

\section{Acknowledgments}

None.

\section{References}

1 Schadendorf D, Lebbé C, Zur Hausen A, et al. Merkel cell carcinoma: epidemiology, prognosis, therapy and unmet medical needs. Eur J Cancer 2017;71:53-69

2 Youlden DR, Soyer HP, Youl PH, Fritschi L, Baade PD. Incidence and survival for Merkel cell carcinoma in Queensland, Australia, 19932010. JAMA Dermatol 2014;150(08):864-872

3 Albores-Saavedra J, Batich K, Chable-Montero F, Sagy N, Schwartz AM, Henson DE. Merkel cell carcinoma demographics, morphology, and survival based on 3870 cases: a population based study. J Cutan Pathol 2010;37(01):20-27

4 Santos-Juanes J, Fernández-Vega I, Fuentes N, et al. Merkel cell carcinoma and Merkel cell polyomavirus: a systematic review and meta-analysis. Br J Dermatol 2015;173(01):42-49

5 Kaufman HL, Russell J, Hamid O, et al. Avelumab in patients with chemotherapy-refractory metastatic Merkel cell carcinoma: a multicentre, single-group, open-label, phase 2 trial. Lancet Oncol 2016;17(10):1374-1385
6 Cowey CL, Mahnke L, Espirito J, Helwig C, Oksen D, Bharmal M. Real-world treatment outcomes in patients with metastatic Merkel cell carcinoma treated with chemotherapy in the USA. Future Oncol 2017;13(19):1699-1710

7 Voog E, Biron P, Martin J-P, Blay J-Y. Chemotherapy for patients with locally advanced or metastatic Merkel cell carcinoma. Cancer 1999;85(12):2589-2595

8 D’Angelo SP, Russell J, Lebbé C, et al. Efficacy and safety of first-line avelumab treatment in patients with stage IV metastatic Merkel cell carcinoma: a preplanned interim analysis of a clinical trial. JAMA Oncol 2018;4(09):e180077. Doi: 10.1001/jamaoncol.2018.0077

9 D'Angelo SP, Bhatia S, Brohl AS, et al. Avelumab in patients with previously treated metastatic Merkel cell carcinoma: long-term data and biomarker analyses from the single-arm phase 2 JAVELIN Merkel 200 trial. J Immunother Cancer 2020;8(01):e000674. Doi: 10.1136/jitc-2020-000674

10 O'Brien T, Power DG. Metastatic Merkel-cell carcinoma: the dawn of a new era. BMJ Case Rep 2018;2018:bcr-2018-224924

11 Kaufman HL, Russell JS, Hamid O, et al. Updated efficacy of avelumab in patients with previously treated metastatic Merkel cell carcinoma after $\geq 1$ year of follow-up: JAVELIN Merkel 200, a phase 2 clinical trial. J Immunother Cancer 2018;6(01):7. Doi: 10.1186/s40425-017-0310-x

12 Nghiem P, Bhatia S, Lipson EJ, et al. Durable tumor regression and overall survival in patients with advanced Merkel cell carcinoma receiving pembrolizumab as first-line therapy. J Clin Oncol 2019; 37(09):693-702

13 Novakovic AM, Wilkins JJ, Dai H, et al. Changing body weightbased dosing to a flat dose for avelumab in metastatic Merkel cell and advanced urothelial carcinoma. Clin Pharmacol Ther 2020; 107(03):588-596

14 Kok DL, Wang A, Xu W, et al. The changing paradigm of managing Merkel cell carcinoma in Australia: an expert commentary. Asia Pac J Clin Oncol 2020;16(06):312-319 